\title{
Molecular Identification of a New Pestivirus Associated with Increased Mortality in the Pyrenean Chamois (Rupicapra pyrenaica pyrenaica) in Spain
}

\begin{abstract}
Ana Hurtado, ${ }^{1,3}$ Gorka Aduriz, ${ }^{1}$ Nieves Gómez, ${ }^{1}$ Beatriz Oporto, ${ }^{1}$ Ramón A. Juste, ${ }^{1}$ Santiago Lavin, ${ }^{2}$ Jorge R. Lopez-Olvera, ${ }^{2}$ and Ignasi Marco ${ }^{2}{ }^{1}$ Department of Animal Health, Instituto Vasco de Investigación y Desarrollo Agrario Neiker, Berreaga 1, 48160 Derio, Bizkaia, Spain; ${ }^{2}$ Servei d'Ecopatologia de Fauna Salvatge, Facultat de Veterinaria, Universitat Autonoma de Barcelona, 08193-Bellaterra, Spain; ${ }^{3}$ Corresponding author (email: ahurtado@neiker.net)
\end{abstract}

ABSTRACT: Pestivirus infection was identified in 16 of 17 chamois during an outbreak of a previously unreported disease in Pyrenean chamois (Rupicapra pyrenaica pyrenaica) in northeastern Spain in 2001-02. By analysis of the $5^{\prime}$ noncoding regions of the virus, we assigned it to the border disease virus cluster with pairwise similarity values ranging from $82.1 \%$ to $88.1 \%$. It will be important to investigate the association of this pestivirus with disease in Pyrenean chamois.

Key words: Border disease virus, chamois, pestivirus, reverse transcriptase polymerase chain reaction, Rupicapra pyrenaica.

The genus Pestivirus belongs to the family Flaviviridae and four main genotypes are recognized by sequence analysis: bovine viral diarrhea virus types 1 and 2 (BVDV-1 and BVDV-2), border disease virus (BDV) of sheep, and classical swine fever virus (CSFV). These genotypes can be further subdivided into subtypes (Becher et al., 1999; Paton et al., 2000; Vilcek et al., 2001; Beer et al., 2002; Hurtado et al., 2003); several pestivirus strains have been described in wildlife as separate groups (Fischer et al., 1998; Becher et al., 1999). The genome of pestiviruses consists of a single, positive stranded RNA molecule that comprises a long, single open reading frame and two noncoding regions (NCRs) at the $5^{\prime}$ and $3^{\prime}$ ends. The $5^{\prime} \mathrm{NCR}$ consists of highly conserved regions useful for polymerase chain reaction (PCR)-based diagnosis of pestiviruses from a wide range of hosts, intercalated by three variable regions (Deng and Brock, 1993) where nucleotide substitutions accounting for differences among pestivirus types are located.

Pestivirus infections in domestic live- stock can cause a variety of economically important syndromes (Houe, 1999), including reproductive failure, poor development, enteric disease, and a hemorrhagic syndrome. Fetal infection can lead to birth of persistently infected (PI) animals that become chronic shedders of the virus. In goats, natural infections with pestiviruses do not appear to be a problem because field cases of clinical disease are rare. Infection of pregnant goats may produce severe placentitis and high death rates among fetuses; few PI kids are born alive after maternal infection (Depner et al., 1991; Lonken et al., 1991; Pratelli et al., 1999).

An outbreak of a previously unreported disease was detected in Pyrenean chamois (Rupicapra pyrenaica pyrenaica) in Catalonia (northeastern Spain) between February 2001 and May 2002. Animals were found within 11,000 ha in the National Hunting Reserve of Alt Pallars $\left(42^{\circ} 35^{\prime} \mathrm{N}\right.$, $1^{\circ} 20^{\prime} \mathrm{E}$ ). Mortality was difficult to evaluate because of landscape characteristics, but a decrease in the population was observed during the annual census. In 2000, before the disease was detected, the population was estimated to be 3,880 chamois and at the end of 2002, the population dropped to 2,678 . The aim of this study was to identify and characterize a pestivirus found in diseased chamois.

Seventeen Pyrenean chamois were captured by hand, culled, or found dead. Ages ranged from $1 \mathrm{yr}$ to $11 \mathrm{yr}$ of age (three females and 14 males). Blood, serum, spleen, or kidney samples, or a combination of these, were collected and analyzed. 
TABLE 1. Results of tests for pestivirus antigens and antibodies in sera and tissues of Pyrenean chamois from Spain during 2001-02.

\begin{tabular}{|c|c|c|c|c|c|}
\hline Chamois no. & $\mathrm{Sex}^{\mathrm{a}}$ & $\begin{array}{l}\text { Age } \\
\text { (yr) }\end{array}$ & $\begin{array}{c}\mathrm{Ab}^{\mathrm{b}} \\
(\text { serum })\end{array}$ & $\begin{array}{c}\mathrm{Agc}^{\mathrm{c}} \\
\text { (sample) }\end{array}$ & $\begin{array}{c}\mathrm{PCR}^{\mathrm{d}} \\
\text { (sample) }\end{array}$ \\
\hline RP-1/01 & M & 3 & Neg & Pos (ser) & ND \\
\hline RP-2/01 & M & 7 & ND & Pos (spl) & Neg (spl) \\
\hline RP-3/01 & $\mathrm{F}$ & 4 & Neg & Pos (ser) & ND \\
\hline RP-4/01 & M & 10 & Neg & Pos (ser) & ND \\
\hline RP-5/01 & $\mathrm{F}$ & 9 & Neg & Pos (ser) & ND \\
\hline RP-7/01 & M & 2 & ND & Pos (spl) & Pos (spl) \\
\hline RP-8/01 & M & 1 & Neg & Pos (ser) & ND \\
\hline RP-1/02 & M & 8 & Neg & Pos (ser) & Neg (kid) \\
\hline $\mathrm{RP}-3 / 02^{\mathrm{e}}$ & M & 9 & Neg & Pos (ser) & Pos (spl) \\
\hline RP-4/02 & M & 8 & ND & Neg (spl) & Neg (spl) \\
\hline$R P-5 / 02^{f}$ & M & 11 & $\mathrm{Neg}$ & Pos (ser) & Pos (spl) \\
\hline RP-6/02 & M & Adult & ND & Pos (spl) & Neg (spl) \\
\hline $\mathrm{RP}-8 / 02^{\mathrm{e}}$ & M & 5 & Neg & Pos (ser) & Pos (spl) \\
\hline RP-9/02 & M & 7 & Neg & Pos (ser) & $\operatorname{Pos}(\mathrm{spl}, \mathrm{bl})$ \\
\hline RP-10/02 & M & 5 & Neg & Pos (ser) & $\operatorname{Pos}{ }^{g}(b l)$ \\
\hline RP-11/02 & M & 8 & Neg & Pos (ser) & Pos (spl, bl) \\
\hline $\mathrm{RP}-12 / 02^{\mathrm{e}, \mathrm{f}, \mathrm{h}}$ & $\mathrm{F}$ & 4 & Neg & Pos (ser) & $\operatorname{Pos} g(\mathrm{bl})$ \\
\hline
\end{tabular}

a $\mathbf{M}=$ male; $\mathbf{F}=$ female

b Tests for serum antibody by blocking enzyme-linked immunosorbent assay (ELISA). Neg = no antibody detected; ND = not done.

${ }^{\mathrm{c}}$ Tests for pestivirus antigen by sandwich ELISA. Pos = samples positive for pestivirus antigen; Neg = negative for pestivirus antigen; ser = serum; spl = spleen

${ }^{\mathrm{d}} \mathrm{PCR}=$ polymerase chain reaction. $\mathrm{ND}=$ not done; $\mathrm{Neg}=$ negative; $\mathrm{Pos}=$ positive; $\mathbf{s p l}=$ spleen; $\mathrm{kid}=\mathrm{kidney} ; \mathrm{bl}=$ blood.

e PCR positive for Anaplasma phagocytophilum.

${ }^{\mathrm{f}} \mathrm{PCR}$ positive to piroplasm.

g $5^{\prime}$ noncoding region of the pestivirus sequenced.

${ }^{\mathrm{h}}$ Results were consistent at five time points (see text).

One animal (RP12/02) was kept alive in captivity for more than $1 \mathrm{mo}$ and blood and serum samples were taken five times during this period (Table 1). Tests were conducted to detect piroplasms by PCR according to Georges et al. (2001) and for Anaplasma by following the methods of Pusterla et al. (2000). Serologic analyses were performed on 13 sera by a blocking enzyme-linked immunosorbent assay (ELISA, Synbiotics, Lyon, France) that specifically detects antibodies directed against a protein (p80/125) common to all strains of BVDV and BDV. Sera or tissue homogenates also were tested for pestivirus antigen by the sandwich ELISA antigen test (Synbiotics). Immunohistochemistry (IHC) on formalin-fixed, paraffin-embedded spleen and kidney samples was conducted by using the monoclonal anti-pestivirus antibody 15C5 directed against the
48-kDa glycoprotein of BVDV (Haines et al., 1992). Amplification of viral RNA by PCR with panpestivirus primers 324/326 was conducted as previously described (Hurtado et al., 2003). The amplified DNA fragments from two animals were purified and both strands were sequenced (285 base pairs [bp]) as described by Hurtado et al. (2003) to identify the pestivirus genotype. Serum samples also were tested for Brucella antibodies by the rose bengal agglutination and complement fixation tests and maedi/ visna antibodies by agar gel immunodiffusion (AGID) (Office International des Epizooties, 2000). The GenBank database (www.ncbi.nlm.nih.gov/Genbank/index. html) was searched for related sequences and multiple sequence alignments were performed using the program AlignX (vector NTI8.0 Suite, InforMax, North Bethesda, Maryland, USA) with an engine 
based on the Clustal W algorithm (Thompson et al., 1994). Alignments were visually checked to take into account secondary structures in the $5^{\prime} \mathrm{NCR}$ and the aligned sequence data were used to construct a phylogenetic tree. Similarity matrices were constructed from aligned sequence data (242 positions compared) by single distance by using the Jukes and Cantor correction for multiple base changes (Jukes and Cantor, 1969). The phylogenetic tree was constructed by the neighbor-joining method (Saitou and Nei, 1987) as implemented in MEGA version 2.1 (Kumar et al., 2001).

Affected chamois had nonspecific signs, such as difficulty moving and absence of flight reaction due to human presence. They were cachectic and had pallid mucous membranes. Eleven chamois had varying degrees of alopecia, mainly of the trunk and neck, with skin hyperpigmentation. All chamois had lower red blood cell counts, hematocrits, and hemoglobin values, compared to reference values (unpubl. data). Piroplasms were detected in two chamois and three were PCR positive for Anaplasma phagocytophilum (Table 1). Serum samples were negative for antibodies to Brucella and maedi/visna viruses.

None of the chamois had antibodies against pestivirus (Table 1). However, all but one was positive for pestivirus antigen on the sandwich ELISA antigen test. Seven of eight spleen and kidney tissue samples were positive for pestivirus antigen by IHC. Immunolabeling was primarily in epithelial cells of the glomeruli and in the low columnar epithelium of proximal tubules where the degree of autolysis was less. Autolysis precluded histologic evaluation of spleen. Amplification of viral RNA by PCR with panpestivirus primers 324/ 326 generated a single band of the expected size $(\sim 290 \mathrm{bp})$ from eight animals (Table 1). Enzyme-linked immunosorbent assay and PCR results of samples from the chamois (RP-12/02) kept in captivity were consistently positive for BVDV at the five time points when samples were collect.
The presence of pestivirus was demonstrated by antigen-capture ELISA, PCR amplification, IHC, or a combination of these in 16 chamois. Lack of PCR amplification and positive IHC staining in a few samples probably was due to sample degradation. Absence of antibodies in all serum samples could have been due to recent infections; however, absence of antibodies in the animal kept alive longer than 1 mo might mean that this was a PI animal. However, it is not known if chamois can become persistently infected with pestiviruses and how long a PI chamois would live.

Two hypotheses should be considered. One hypothesis is that the pestivirus infection appeared several years previously, but remained undetected until it spread throughout a large part of the population. This hypothesis is supported by evidence of persistent viremia in RP-12/02. Alternatively, the disease could have been the result of introduction of a virulent pathogen with an efficient transmission rate. This would be supported by lack of antibodies in the animals tested and occurrence of overt disease. The lack of immune response to the pestivirus infection could have been due to coinfection with Theileria, Anaplasma, or both, or immunosuppression due to scarce food availability and poor condition in the winter period. However, the presence of a pestivirus and absence of antibody production requires additional investigation.

The amplified DNA fragments from two animals (Table 1) consisted of $285 \mathrm{bp}$ and were identical and henceforth regarded as belonging to the same strain designated as Chamois-Spain02, and deposited in GenBank under accession number AY641529. The chamois strain formed a separate branch within the BDV cluster (Fig. 1). Pairwise comparisons showed highest similarity values (88.1-87.2\%) with BDV subtype A strains (accession numbers U65036 and U65048, respectively). Similar values (88.1-86.9\%) were obtained between Chamois-Spain02 and BDV sub- 


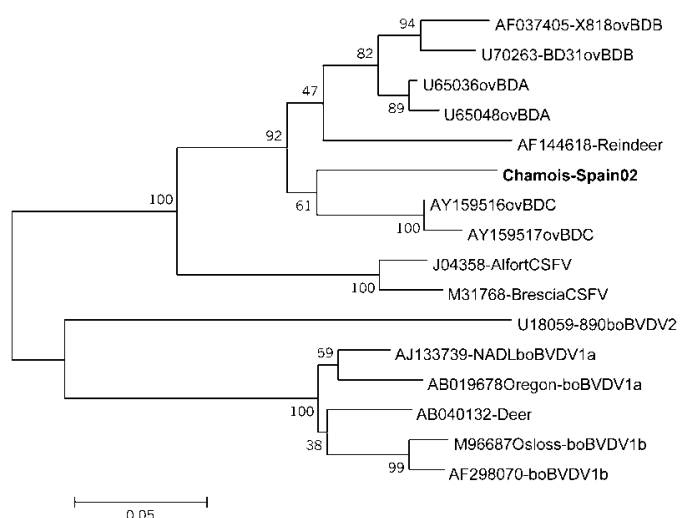

FIGURE 1. Neighbor-joining phylogenetic tree of the $5^{\prime}$ noncoding region of a strain of pestivirus from Pyrenean chamois in Spain during 2001-02. To test the reliability of the branches in the tree, a bootstrap analysis (1,000 replicates) was performed by creating a series of bootstrap samples; obtained values are shown on each branch. The samples have been coded with the GenBank accession number, strain (if a reference strain), host of origin ( $b o=b o v i n e ; ~ o v=o v i n e)$, and genotype. The length of horizontal branches reflects phylogenetic distance as indicated by the scale below.

type C strains (AY159516 and AY159517), whereas similarity with BDV subtype B (AF037405 and U70263) was slightly lower (84.3-82.1\%, respectively). The nearest wild ungulate strain to Chamois-Spain02 was isolated from a reindeer (Rangifer tarandus) (strain V60-Krefeld, AF 144618) and showed $83.0 \%$ similarity by pairwise comparison analysis. Bootstrap analysis confirmed a high confidence $(>90 \%)$ in the groupings of the genotypes, thus supporting the absolute position of ChamoisSpain02 strain within the BDV cluster. However, although in the tree shown in Figure 1 Chamois-Spain02 clusters with BDV subtype $\mathrm{C}$, this branching showed a low confidence level $(61 \%)$ so that the absolute position of the chamois strain within the BDV subtypes could not be clearly defined.

Harasawa and Giangaspero (1998) reported a method for pestivirus genotyping based on secondary structure analysis of the three variable $(\mathrm{V})$ domains of the 5 'NCR. Secondary structures with the lowest free energy as predicted by Zucker's algorithm (Jeager et al., 1989) revealed that V1 and V2 of Chamois-Spain02 strain were highly similar to BDV strains, whereas V3 was clearly different from all described genotypes (data not shown). The $\mathrm{V} 3$ region has been reported to be of different size in different genotypes (Harasawa and Giangaspero, 1998), being shorter in BDV and CSFV genotypes (16 and 15 nucleotides, respectively) than in BVDV-1 and BVDV-2 (19 or 20, and 18 nucleotides, respectively). The V3 region of Chamois-Spain02 strain is 16 nucleotides in length, as it is in BDV strains. However, the stem-loop structure of Chamois-Spain02 strain is different from that of all other genotypes. The stem structure of BVDV-1 and BVDV-2 consists of $7 \mathrm{bp}$, and of $6 \mathrm{bp}$ in BDV and CSFV, whereas the Chamois-Spain02 strain has a 4-bp stem with an A:C bulge in position 2. The loop of the $\mathrm{V} 3$ region consists of three nucleotides in CSFV, four nucleotides in BDV and BVDV-2, and five or six nucleotides in BVDV-1. In ChamoisSpain02 strain, however, the loop consists of six nucleotides.

Regardless of being considered a new subtype or not, pestiviruses seem to have a high rate of mutation and the ability to infect different hosts such as Pyrenean chamois. Nucleotide substitutions within the $5^{\prime}$ NCR have been used to distinguish high-virulence from low-virulence BVDV2 strains (Topliff and Kelling, 1998). Further studies are needed to determine virulence markers associated with other pestiviruses such as the one reported here.

An increasing number of pestiviruses are being identified by molecular techniques in different hosts, but pestivirus infection has not been associated with disease in Pyrenean chamois previously. Chamois appear to be highly susceptible to infection with this pestivirus. A single affected animal was reported in the study area after the 2001-02 outbreak, and the same pestivirus was identified by sequence analysis. Studying additional cases of pestivirus infections may allow us to under- 
stand how the Pyrenean chamois reacts to this virus and what effect the virus may have on chamois populations.

Financial support was provided by the Departament de Medi Ambient of the Catalonia Government. We are grateful to the director and wardens of the National Hunting Reserve of Alt Pallars for assisting in the capture of the chamois, to E. J. Dubovi for providing monoclonal anti-pestivirus antibody 15C5, and E. Berriatua for comments and critical discussion.

\section{LITERATURE CITED}

Becher, P., M. Orlich, A. Kosmidou, M. Konig, M. Baroth, and H. J. Thiel. 1999. Genetic diversity of pestiviruses: Identification of novel groups and implications for classification. Virology 262: 64-71.

Beer, M., G. Wolf, and O. R. KaAden. 2002. Phylogenetic analysis of the $5^{\prime}$-untranslated region of German BVDV type II isolates. Journal of Veterinary Medicine B 49: 43-47.

DENG, R., AND K. V. BROCK. 1993. $5^{\prime}$ and $3^{\prime}$ untranslated regions of pestivirus genome: Primary and secondary structure analyses. Nucleic Acids Research 21: 1949-1957.

Depner, K., O. J. Hubschle, and B. Liess. 1991. BVD-virus infection in goats: Experimental studies on transplacental transmissibility of the virus and its effect on reproduction. Archives of Virology. Supplementum 3: 253-256.

Fischer, S., E. WeILAND, AND K. Frölich. 1998. Characterization of a bovine viral diarrhea virus isolated from roe deer in Germany. Journal of Wildlife Diseases 34: 47-55.

Georges, K., G. R. Loria, S. Ritli, A. Greco, S. Caracappa, F. Jongejan, and O. Sparagano. 2001. Detection of haemoparasites in cattle by reverse line blot hybridization with a note on the distribution of ticks in Sicily. Veterinary Parasitology 99: 273-286.

Haines, D. M., E. G. Clark, and E. J. Dubovi. 1992. Monoclonal antibody-based immunohistochemical detection of bovine viral diarrhea virus in formalin-fixed, paraffin-embedded tissues. Veterinary Pathology 29: 27-32.

Harasawa, R., AND M. Giangaspero. 1998. A novel method for pestivirus genotyping based on palindromic nucleotide substitutions in the $5^{\prime}$ untranslated region. Journal of Virological Methods 70: 225-230.

Houe, H. 1999. Epidemiological features and economical importance of bovine virus diarrhea virus (BVDV) infections. Veterinary Microbiology 64: 89-107.

Hurtado, A., A. L. Garcia-Perez, G. Aduriz, AND
R. A. Juste. 2003. Genetic diversity of ruminant pestiviruses from Spain. Virus Research 92: 6773.

Jeager, J. A., D. H. Turner, And M. Zuker. 1989. Improved predictions of secondary structures for RNA. Proceedings of the National Academy of Sciences of the USA 86: 7706-7710.

Jukes, T. H., AND C. R. CANTOR. 1969. Evolution of protein molecules. In Mammalian protein metabolism, H. N. Munro (ed.). Academic Press, New York, New York, pp. 21-132.

Kumar, S., K. Tamura, I. B. Jakobsen, and M. Nei. 2001. MEGA2: Molecular evolutionary genetics analysis software. Arizona State University, Tempe, Arizona.

Loken, T., J. Krogsrud, and I. BJerkas. 1991. Outbreaks of border disease in goats induced by a pestivirus-contaminated orf vaccine, with virus transmission to sheep and cattle. Journal of Comparative Pathology 104: 195-209.

OFFICE INTERNATIONAL DES EPIZOOTIES. 2000 Manual of standards for diagnostic tests and vaccines. Paris, France, pp. 328-345, 497-502.

Paton, D. J., A. McGoldrick, I. Greiser-Wilke, S. Parchariyanon, J. Y. SONG, P. P. Liou, T. STADEjEK, J. P. LOWINGS, H. BJORKLUND, AND S. BELAK. 2000. Genetic typing of classical swine fever virus. Veterinary Microbiology 73: 137-157.

Pratelli, A., E. Bollo, V. Martella, F. Guarda, D. Chiocco, and C. Buonavoglia. 1999. Pestivirus infection in small ruminants: Virological and histopathological findings. New Microbiology 22: 351-356.

Pusterla, N., J. S. Chae, E. Derock, and J. E. Madigan. 2000. One-tube PCR for the detection of the Ehrlichia phagocytophila genogroup. The Veterinary Record 147: 22-23.

Saitou, N., AND M. NEI. 1987. The neighbor-joining method: A new method for reconstructing phylogenetic trees. Molecular Biology and Evolution 4: $406-425$.

Thompson, J. D., D. G. Higgins, and T. J. Gibson. 1994. CLUSTAL W: Improving the sensitivity of progressive multiple sequence alignment through sequence weighting, position-specific gap penalties and weight matrix choice. Nucleic Acids Research 22: 4673-4680.

ToplifF, C. L., AND C. L. Kelling. 1998. Virulence markers in the $5^{\prime}$ untranslated region of genotype 2 bovine viral diarrhea virus isolates. Virology 250: 164-172.

Vilcek, S., D. J. Paton, B. Durkovic, L. Strojny, G. Ibata, A. Moussa, A. LoItsch, W. RossmanITH, S. Vega, M. T. SCICluna, AND V. PAIfI 2001. Bovine viral diarrhoea virus genotype 1 can be separated into at least eleven genetic groups. Archives of Virology 146: 99-115.

Received for publication 7 November 2003. 